Buychik, A. (2020). The Theory of Wave Dualism of Cracy as a tool for modeling various processes in society. Past and present in the philosophy of social development. European Scientific e-Journal, 2 (2), 33-49. Hlučín-Bobrovníky: "Anisiia Tomanek" OSVČ.

DOI: $10.47451 /$ pol2020-10-001

The paper will be published in Crossref, ICI Copernicus, Academic Resource Index ResearchBib, J-Gate, ISI International Scientific Indexing, Zenodo, OpenAIRE, BASE, LORY, LUASA, ADL, eLibrary, and WebArchive databases.

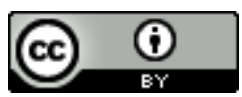

Alexander Buychik, Doctor of Economic Sciences, PhD of Social and Political Sciences, Chief Director, Tuculart Scientific Holding. Ostrava, Czech Republic.

\title{
The Theory of Wave Dualism of Cracy as a tool for modeling various processes in
} society

Abstract: At the turn of the 20th and 21st centuries, there was an urgent need to measure the political activity of certain elites, which determine the direction of development of society both within the state and in the international arena. Political elites of society set up certain markers that allow them to create the necessary social background in the state for the purpose of lobbying interests. The author presents basic principles of the Theory of Wave Dualism of Cracy (Power), which are design for modeling and forecasting political, social, economic and cultural processes in the society. The theory consists of general and specific terms parameters that have multi-level characteristics, which can contribute to more correct verification of processes. The author concludes that Theory of Wave Dualism of Cracy forms a more objective approach to historical processes and events in various fields of society.

Keywords: dualism, power, political processes, political event, Second World War, postulation, classification of political processes, social matrix.

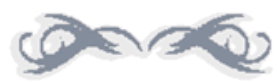

\section{Introduction}

At the turn of the 20 th and 21 st centuries, there was an urgent need to measure the political activity of certain elites, which determine the direction of development of society both within the state and in the international arena. Political elites of society set up certain markers that allow them to create the necessary social background in the state for the purpose of lobbying interests.

To describe the behavior patterns of political elites, it is no longer enough to apply general postulates and axioms expressed in a textual way. As with economic process modeling, it is necessary to create models that apply both political laws and active data systems to describe political processes. Numerous studies of leading physicists in the field of cognition of the surrounding space served as an impetus to rethink the processes occurring in social strata and cultures.

As a result, the need to update the issue of complex modeling of complex political processes has increased progressively in the early 21 st century. The issue of modeling a political personality as a leader or representative of the vanguard of a political movement or political party was also updated. A political personality in the modern information society performs not only the functions of a conductor of the social and political concept of the elite that formed or promoted 
him or her to leadership positions, but such a person is already a certain attribute of power, often eclipsing the elite or replacing it in a number of ideas about the political values of a party or political movement.

In the course of this research, a comprehensive analysis of power as a phenomenon of development of modern society was made from the point of view of creating a basis for modeling, the purpose of which is to predict political processes and identify the primary sources of their origin.

To explain the application of modeling to the characterization of political processes, their genesis and variability of development, the Theory of Wave Dualism of Cracy (TWDC) was created. Power, or cracy, in any form of its manifestation has existed for hundreds of thousands of years. It was born in the era of ancient intelligent man and constantly developed throughout the evolution of society to the era of Modern Man in the framework of the evolution period of Homo sapiens sapiens. Power does not so much control an individual or a group of individuals, as it exerts a purposeful influence on the consciousness and place in the environment of the person being affected.

Since power is eternal in the understanding of human society, its primary source is the desire to realize the potential and the need to manage the environment. In the global sense, power is changeable, periodic in its manifestations and excludes temporal stagnation. Thus, there is a need for a comprehensive analysis of power as a phenomenon of human society development by modeling and forecasting all types of political processes taking into account a large number of initial data contained in a certain set of markers.

The purpose of the research is to create basic concepts and markers for modeling a single set of political, economic, social and cultural processes at the national, regional and international levels based on the characteristics of the dominant power and the interaction of various democracies, based on its specific postulates.

Based on the purpose of the study, the following tasks were set:

1. Determine the structure of concepts and elements of the complex of social, political, economic and cultural processes, which will allow to present the trends of the situation in sufficient detail and schematically.

2. Confirm the wave principle of development of processes' complex.

3. Confirm the dualistic principle of influence of any government on the complex of processes.

4. Create a method for predicting the development of various processes occurring in society accurately enough for its further experimental use.

\section{Postulating within the framework of the Theory of Wave Dualism of Cracy}

A postulate is a statement that is taken to be true, to serve as a premise or starting point for further reasoning and arguments (Postulate, 2010). The term has some differences in definition when used in the context of different fields of study. According to Aristotle's Posterior Analytics, a postulate, or axiom, is a statement that is so evident or well-established, that it is accepted without controversy or question (Mure, 2007). Also, we can used modern logic term that a postulate, or axiom, is a premise or starting point for reasoning (Axiom, 2015).

Within the framework of the Theory of Wave Dualism of Cracy, five postulates are put forward: 
Postulate 1. The characteristic of power directly depends on the complex intelligence coefficient of the political leader of the state or the subject of the state. This postulate is derived from a practical comparison of the share of political leaders, who formed the political sphere in the state quite independently after being elected or coming to power through a coup d'etat by displacing one elite to another, from the total number of political leaders in Europe, America and the leading Asian states.

Postulate 2. Power forms a matrix, i.e., a complex of political, social, economic, and cultural processes. Power cannot exist outside of society; it takes the initial parameters and characteristics of society and then makes the necessary transformations subordinating society through the formation of a set of planned processes in all spheres of society. This is how the government solves two main tasks - its leading position and lobbying for public values that ensure this leadership, which is already distinguished by its quasi-natural origin.

Postulate 3. Power evolves in waves in a temporal coordinate system, where geographical location is of secondary importance. Political elites, returning to power as a result of elections or a coup d'etat, inevitably transform their previous experience and adapt it to the new realities of the social, political and economic state of society. As a result, there is inevitably an evolution of power. However, this evolution is not a revolution, but retains a significant share of its political content, which is the basis of this political elite. Consequently, there is a wave process of power evolution.

Postulate 4. Power in any form and any of its manifestations is dualistic in the eyes of society or community. Based on the concept of dualism, we must recognize that the nature of power is differently perceived by people. Given the understanding that everything in nature has action and reaction, we expect and observe two opposite views of representatives of society on any power, i.e., those who agree with the power and those who disagree with the power. In this case, agreement and disagreement are identical to the essence of understanding the criteria and values of power, i.e., its dualistic nature in the eyes of society in general.

Postulate 5. All events that occur in society have multiple historical identity. Any political processes that take place in history have analogs in the temporal sense. Analog means not a copy, but a certain identity, i.e., the common nature of the process itself with processes that occurred in the past. In the course of the development of society, methods and tools for implementing any concepts are improved, but the concepts of power themselves, implemented through political processes, have ancient origins that are preserved to this day.

Postulating a political theory is an extremely important stage in its formation and development. Without a basis, we cannot build models for the implementation of political processes and formulate sufficiently realistic variations in the forecast of their development in the future. It is the multiplicity of variations that have a common logical basis that will be valuable and help to develop correct methods for implementing or not implementing political processes.

Thus, the postulation of political theory allows us to get a valuable basis for the correct creation of the categorical apparatus and methodology. Regarding the Theory of Wave Dualism of Cracy, the postulation allowed us to define general and specialized terms, confirm the components of the political system without taking into account its diversity, and create a correct classification of specialized characteristics, the terms of which are already defined. 


\section{Terms used in the TWDC and their definition}

As part of the development of the Theory, a terminology dictionary, which is mandatory for description, was defined as help to appeal to properties used in theory.

The general terms necessary to describe the theory were defined as follows:

Cracy, or power, is the capacity of a person to influence the actions, beliefs, or conduct of others. The term authority is often used for power that is perceived as legitimate by the social structure. In general, it is derived by the factors of interdependence between two entities and the environment (Schein \& Greiner, 1988). The concept of cracy is key in the Theory, since it first forms a political personality and then is formed according to the political personality that has reached a certain peak of it, i.e., received the its tools.

$W$ ave is a change in the state of a medium that propagates in this medium and carries energy (Gorelick, 1959). Within the framework of the Theory, the wave takes on a similar meaning in relation to political processes, i.e., a change in the political state of the social environment that spreads in this environment and carries with itself political processes that have a certain impact on this environment.

Dualism is a phenomenon that implies the dual nature of a particle. Also, dualism in cybernetics refers to systems or problems, in which one or more intelligent adversaries attempt to exploit the weaknesses of the investigator. In politics, the term dualism is used to refer to the separation of powers between the cabinet and parliament. Within the framework of the Theory, dualism is used as a duality of the nature of perception of wave processes of evolution or development of power.

Dichotomy is division in two (Komjath \&otik, 2006), in logic it is division into two rows. Within the framework of the Theory, this term is used in the general understanding of the division of political power and political processes into two entities represented by opposite views.

Verification is a concept used in the logic and methodology of scientific knowledge to refer to the process of establishing the truth of scientific statements through their empirical verification (Global Harmonization Task Force, 2004). Verification consists in correlating the statement with the actual state of Affairs by observation or measurement. The term 'verification' often is changed by the term 'validation', but in practice, as quality management terms, the definitions of verification and validation can be inconsistent. Sometimes they are even used interchangeably (McCaffrey, 2006). Checking the truth of theoretical statements and establishing their validity is fundamental in the practical application of theoretical developments. Therefore, if we confirm the correctness of theoretical statements, then an irrefutable truth is already formed in front of us. However, Karl Popper in 1935 put forward a criterion that States that a scientific theory cannot be fundamentally irrefutable. This, according to Popper [1], solves the problem of demarcation, i.e., the separation of scientific knowledge from non-scientific.

Also, the Theory consists of some special terms, which was generated from other sciences as analog concepts in help to understanding of the evolution of cracy and political processes:

Matrix (M) is the state of the political system of a state or states' association at the time of analysis or the analyzed time period. The political system has a set of characteristics that can be enclosed in Matrix. In this case, the set of characteristics becomes the most stable and can contribute to the correct taxonomy with a variety of such features and get a certain place in it. 
Volumeness $(\mathrm{Vol})$ is the percentage size of individuals to the total number of representatives of a society or community. Within the framework of the Theory, individuals are a special part of society that has a high level of intelligence and consciousness that distinguishes them from the rest. Individuals will be personnel who exceeded the qualification standard of education in the country (usually master and higher), researchers and creative people highlighted in the state or international community (awards for contribution to the development of society, science, culture and art).

Valency (Val) is the level or price of Volumeness. Within the framework of the Theory, value gains special weight, since the price of individuals in society is an important component. The price of individuals is highest in a truly democratic society, where there is no censorship of the opposition society. In 2020, the leading countries of the world - G-8 - do not have such a high level of democratization of society. This is due to the growing political tension of elites, which strive to control the opinion of individuals. This control generates political inertia of the majority of individuals or extreme caution in the public expression of opinions. The same effect is being observed more and more in other states of G-20. Therefore, Valency correctly specifies Volumeness.

Quest (Q) is an event of various types - political, social, economic, cultural. An event is the main component of temporality. It shapes history. The sequence of events forms a sequence that we often call a chain of events. It is impossible to assess the temporal development of political processes without analyzing not only each event, but also the totality of events, as well as categorizing events by their main characteristics. If we accept each event as unique, we cannot categorize or systematize their set, and therefore we cannot find identity in the past and predict events in the future.

Quasar (Q) is the most remote quest in the historical sense, which characterizes the genesis of the state of the analyzed the Matrix. Finding the root cause is the main problem of philosophy, which turns into a historical problem. The search for the root cause usually does not bring results, because its concept is extremely complex and vague. Each specialist understands the root cause individually, therefore, determining the event that caused the subsequent chain of events remains an unsolved task. However, establishing Quasar is a more meaningful and purposeful action, since it provides for a fundamentally consistent and logical passage through the chain of events of the past moving away from the present and highlighting only the most important thing on the principle of Occam's Razor at each historical stage.

Fragment $(\mathrm{F})$ is a historical fact, an integral element of the Quest. Fragment is not an event. A fact is a truth that is formed by an event or set of events. This fact cannot be ignored or interpreted in any other way. Thus, a fact is an objective and verifiable observation (Gower, 1997). If this statement is correct, then the set of facts is the basis for analyzing any Quest.

Station (S) is a complex characteristic of the analyzed state of society or process. This term is quite objective and does not require special disclosure. It is used to model political processes and the political state of society within the framework of the Theory.

Archion (A) is a complex characteristic of the state of society or the process of a past moment in history, not directly related to the implementation of Station. Archion is the same Station that passed temporally into the category of a past event. With the next change in the characteristics of the society, Station becomes Archion and serves as an analytical material both in general and 
for individual Quests and Fragments. Also, in the future, any archion quest may become a quasar of another quest that is being analyzed.

Version $(\mathrm{V})$ is a complex characteristic of one of the variants of the predicted state of society or process. Version is identical to the matrix. However, it is also identical in its essence to Archion, since it has a multiplicity of options, the number of which is regulated by the correctness of modeling and forecasting.

Relic Background (RB) is a set of consecutive Quests of Archions, which characterize the genesis of the analyzed Quest or the state of the Matrix. It has a set of Quests inside Archions that are farthest from the Matrix in the temporal dimension, but they are the ones that retain the special influence on the Matrix.

Thus, within the framework of the Theory, general and specialized basic terms were developed that describe power and political processes that occur and constantly evolve over time taking into account the basic understanding of the Theory and its postulates. These concepts are of fundamental importance, because they are the most relevant in describing the position of the Theory to the dualistic wave nature of cracy (power) and contain a detailed classification that helps to model and predict the Matrix.

\section{Features of classification of the main parameters}

In order to correctly model and predict the Matrix, a classification of the main parameters of cracy (power) and political processes, which were characterized as terms of the Theory in section 2 of this article, was developed.

(3.1) Quests

Quest (Q) is a reference parameter for modeling political processes, so the classification of quests (events) is of particular importance. Within the framework of the Theory, it is proposed to introduce three classification groups.

Group 1. Classification by percentage size or number of socially dependent individuals:

- Regional Quests (RQ) are events of regional or local importance;

- National Quests (NQ) are events that affect several significant or most regions of the state;

- International Quests (IQ) are events that affect several states;

- Macro Quests (MQ) are events that have continental or global level.

Individuals form the social format of a political event, so their correlation and territorial distribution is one of the key values in determining the characteristics of an event, or Quest. As an example, we can take the political conflicts that later led to military actions. If only indviduals of one state participate in a political event-conflict, it retains the national level (NQ). If at least a small percentage of geographically distinct individuals join the conflict, the event takes on an international character (IQ).

Group 2. Classification by significance level for a specific volumeness:

- macro-quests (macQ) are events where the share of socially independent individuals from the total number of them participating in the event exceeds 50 percent, i.e., their independence prevails in the competent social opinion and can objectively influence the political process within this Quest, 
- meso-quests (mesQ) are events where the share of socially independent individuals from the total number of them participating in the event is from 30 to 50 percent, i.e., their independence influences the competent social opinion in some ways, so they can objectively influence the political process within this quest but such influence is less than in the class of macro-quest;

- micro-quests (micQ) are events where the share of socially independent individuals from the total number of them participating in the event is less 30 percent, i.e., their independence has little influenced the competent social opinion because the main part of individuals depends on the government and suppresses the social position of independent individuals with its majority.

Each of these indicators has its own percentage coefficient, which is calculated from 50 percent of the volume of independent individuals as a single unit (1.00). Therefore, each percentage of this scale is equivalent to 0.02 in the Matrix model.

Group 3. Classification according to the type of genesis event:

- $\quad$ Political Quests (Qp);

- $\quad$ Social Quests (Qs);

- Economical Quests (Qe);

- Cultural Quests (Qc).

These four types of events are the most volume and important, so we should take genesis when modeling the Matrix.

(3.2) Fragments

Fragment cannot be classified within the framework of the Theory, because the fact has a unique characteristic. The only possible interpretation of a fact is that it may be identical to other facts to varying degrees regarding its influence on this Quest. Fragments are part of the "input" of the Quest and form the "output" of the Quest, i.e., the original estimated name of the event, in a harmonious and logical combination. Therefore, Quests are part of the "input" of the Arbion, Station, or Version, and they form the 'output' of Arbion, Station, or Version.

(3.3) Stations

A set of interrelated Quests, or processes that are subject to a holistic analysis, forms different types of Stations. The typology of Stations can be grouped into four groups.

Group 1. Classification by the genesis set of Quests:

- mono-Stations $(\mathrm{mS})$ consist of the same type of genesis Quests;

- full-Stations (fS) consist of all four types of genesis Quests;

- quasi-Stations (qS) consist of Quests of two or three genesis types.

This classification simplifies the interpretation of Quests and clearly shows the variability of their combination when creating Station.

However, according to this classification, the Theory needs to clarify the classification of mono-Stations, so we should offer Goup 2 - classification of mono-Stations by their genesis:

- $\quad$ political (mSp);

- $\quad$ social ( $\mathrm{mSs})$;

- $\quad$ economical (mSe);

- $\quad$ cultural (mSc). 
Also, according to this classification, the Theory needs to clarify the classification of quasiStations, so we should offer Group 3 - classification of quasi-Stations by their genesis:

- $\quad$ socio-political (qSsp);

- $\quad$ socio-economical (qSse);

- $\quad$ socio-cultural (qSsc);

- $\quad$ politic-economical (qSpe);

- $\quad$ politic-cultural (qSpc);

- $\quad$ triple (qSt).

Triple quasi-Stations are complex ones, so we do not need to separate individual sub-types. Group 4. Classification by predicted destination of the Stations:

- stable ones are Stations that have a definite and direct value for the nearest changes (perturbations) of the Matrix, i.e., pre-predicted or officially expected Quests of equal or higher level of Volumeness or Valency;

- unstable ones are Stations that have possible values for the long-term predicted change (perturbation) of the Matrix, i.e., used for constructing Versions.

This classification clearly separates the Stations into those that are necessary for modeling the existing Matrix, and those that will be relevant for constructing the variation of subsequent events, which will form a new Matrix in the future. Station can contain quests of different $V$ olumness, Valency, and genesis that differ from each other, but are interrelated and uniquely define it.

Relevance of Station allocation is:

1) selection of interrelated Quests by genesis;

2) clear classification of the set of Quests distributed fairly densely over time;

3) comprehensive analysis of the selected Quests;

4) development of a method for allocating Stations for a more systematic and detailed analysis of various processes occurring in the Matrix.

(3.4) Versions

A set of interrelated predicted Quests that are subject to a holistic analysis, forms different types of Versions. They are classified in the same way as Stations with the exception of predictive purpose, which is typical for future Quests. Versions are identical to the classification of Stations, since they are the same Stations of the future Matrix.

Group 1. Classification by the genesis set of Quests:

- mono-Versions (mV) consist of the same type of genesis Quests;

- full-Versions (fV) consist of all four types of genesis Quests;

- quasi-Versions (qV) consist of Quests of two or three genesis types.

Goup 2. Classification of mono-Versions by their genesis:

- $\quad$ political $(\mathrm{mVp})$;

- $\quad \operatorname{social}(\mathrm{mVs})$;

- economical (mVe);

- $\quad$ cultural $(\mathrm{mVc})$.

Group 3. Classification of quasi-Versions by their genesis: 
- $\quad$ socio-political (qVsp);

- $\quad$ socio-economical (qVse);

- $\quad$ socio-cultural (qVsc);

- $\quad$ politic-economical (qVpe);

- $\quad$ politic-cultural (qVpc);

- $\quad$ triple $(\mathrm{qVt})$.

Triple quasi-Versions are complex ones, so we do not need to separate individual sub-types. Group 4. Classification according to predicted purpose:

- independent ones are Versions that according to forecasts, will be considered as a complete set of Quests for future changes (perturbations) of the Matrix that leads to its evolution and the formation of new one;

- dependent ones are Versions that according to forecasts, will be considered as conjunction with other possible, genetically designed or required Versions for future changes (perturbations) of the Matrix.

Relevance of Version allocation is:

1) selecting the necessary interconnected Quests based on their genesis and launching the process design based on them;

2) a clear classification of the set of Quests that involve in the design of changes to the existing Matrix and distribute quite tightly on the temporal tape of the model;

3) comprehensive analysis of selected predicted Quests;

4) development of a method to select Versions for a more systematic and detailed analysis of various processes designed for the evolution of the Matrix.

(3.5) Archions

A complex characteristic of the Quests that occurred in the past and is not directly related to Stations, including the Quests that form them, is called an Archion. Archeons no longer directly affect the existing Matrix, i.e., they are so remote that they are not directly related to the concept of 'today's events', 'events of this period'. However, Archeons are also classified in the same way as Versions.

Group 1. Classification by the genesis set of Quests:

- mono-Archions (mA) consist of the same type of genesis Quests;

- full-Archions (fA) consist of all four types of genesis Quests;

- quasi-Archions (qA) consist of Quests of two or three genesis types.

Goup 2. Classification of mono-Archions by their genesis:

- $\quad$ political (mAp);

- $\quad$ social (mAs);

- economical (mAe);

- cultural (mAc).

Group 3. Classification of quasi-Archions by their genesis:

- $\quad$ socio-political (qAsp);

- $\quad$ socio-economical (qAse);

- $\quad$ socio-cultural (qAsc); 
- $\quad$ politic-economical (qApe);

- $\quad$ politic-cultural (qApc);

- $\quad$ triple (qVt).

Triple quasi-Archions are complex ones, so we do not need to separate individual sub-types. Group 4. Classification according to predicted purpose:

- independent ones are Archions that according to Fragments, were considered as a complete set of Quests for past changes (perturbations) of the Matrix that was a result of previous Matrix evolution;

- dependent ones are Archions that according to Fragments, were considered as conjunction with other possible, genetically designed or required Archions for past changes (perturbations) of the Matrix that was a result of previous Matrix evolution.

Relevance of Version allocation is:

1) selecting the necessary interconnected Quests based on their genesis and launching the process design based on them;

2) a clear classification of the set of Quests that involve in the design of changes to the past Matrix and distribute quite tightly on the temporal tape of the model;

3) comprehensive analysis of selected predicted Quests;

4) development of a method to select Archions for a more systematic and detailed analysis of various processes that produced perturbation of the Matrix in the past in order to use them in the system of analysis of Station;

5) development of a method to select Archions for a more systematic and detailed analysis of various processes that produced perturbation of the Matrix in the past in order to use them in the prediction system of the Matrix and modeling of the groups of Versions.

(3.6) Quasar

The most remote Quest in the historical sense, which characterizes the genesis of the analyzed Quest, Archion, Station, or Version, as well as the state of various Matrixes (or evolutionary states of the Matrix) is a Quasar. A quasar Quest is extremely difficult to identify because of a rather large proportion of subjectivism in assessing its significance for the process under consideration or analyzed Archion, Station, or, even more so, Version. In this regard, within the framework of the Theory, Quasars have one type of classification.

- $\quad$ proto-Quasar (pZ) is a Quest that supposedly took place in the relatively or actually distant past, or is particularly significant for determining the corresponding Archions, Stations, or Versions;

- real Quasar ( $\mathrm{rZ}$ ) is a Quest that reliably occurred in the relatively or actually distant past, is relative to the studied event and fundamentally significant to determine the studied event;

- debatable Quasar (dQ) is a quest that reliably occurred in the relative or actually distant past relative to the event being studied, but its significance is highly controversial.

An example is the micro-Quest of the beginning of Second World War. There are two historical versions. The modern European version claims that the Molotov-Ribbentrop Pact was the beginning of the war, after which the occupation of Poland began. The Russian version differs in that the beginning of the war came at the time of the betrayal of Poland by the German elites and the beginning of the seizure of territory. However, if we consider the genesis of the beginning of the 
Second World War in the framework of the Theory, the Quasar of this micro-Quest was the partition of Czechoslovakia. This event (Quest) became the political right of Germany and its allies to divide Czechoslovakia to expand other territories. Regarding the beginning of the Second World War, the unification of Germany and Austria cannot be considered a Quasar, since it had a distinctive genesis.

The importance of allocation of Quasars:

1) search for and find likely primary sources of origin of a certain type of Quest, Archion, or Station;

2) creat a more accurate methodology to model Stations and Versions in the framework of the Theory.

(3.7) Relict Background

The set of Quests that formed Archions and formed a complete analytical picture in relation to the current Matrix, is a Relic Background. It is only valid if all Quests of Arcbions are linked to the existing state of the Matrix. The Relic Background has an 'input' that is represented by a real Quasar that guarantees verification of the entire Relic Background.

Thus, a detailed and structural classification of the main parameters of the Theory helps to more correctly characterize historical events of political, social, economic, and cultural types. Within the framework of the Theory, it is necessary to take into account a clear differentiation of grouped Quests by time into Stations, Archions, and Versions.

\section{Graphical representation of the basic principles of modeling in the framework of the Theory}

Now it is necessary to graphically present the general principles of Matrix modeling according to the Theory. Graphical representation helps to understand the contextual information presented in sections 1-3 of this article, and to describe the main visual representations that are formed within the framework of the Theory [Fig. 1-3].

Thus, the graphical representation of the basic principles of modeling the Matrix and sequence of events shows a clear construction of historical events, which minimizes distortions and dualities of judgments. Modeling is done in a complex combination of Quests inside Archions, Stations, and Versions. Formed Fragments should be included in them at the input and formed inside, which remain at the output.

\section{Conclusion}

Theory of Wave Dualism of Cracy forms a more objective approach to historical processes and events in various fields of society. This increased correctness is achieved by a fundamentally different approach to the systematization of events, facts, and processes, which is based on a multi-level classification that takes into account time, relative, and absolute parameters. This approach allows us to take a different look at historical processes and justify their genesis. This approach also forms a completely different principle of modeling and forecasting events.

This modeling can be applied to substantiate political, social, economic, and cultural processes, as well as to create a specific set of probable changes in the state of society, taking 
into account both new parameters of the Matrix state and preserved, relatively constant parameters of the past.

\section{0 \\ References:}

Axiom (2015, June 18). Oxford English Dictionary Retrieved May 6, 2020, from https://www.oxfordlearnersdictionaries.com/definition/english/axiom

Global Harmonization Task Force - Quality Management Systems - Process Validation Guidance (GHTF/SG3/N99-10:2004 (Edition 2), page 301.

Gorelick, G.S. (1959). Vibrations and waves. Introduction to acoustics, Radiophysics, and optics. Moscow: Gosizdat. (in Russian)

Gower, B. (1997). Scientific Method: A Historical and Philosophical Introduction. Routledge.

Komjath, P., \& Totik, V. (2006). Problems and Theorems in Classical Set Theory. Springer Science \& Business Media.

McCaffrey, J.D. (2006, April 28). Validation vs. Verification. Software Research, Development, Testing, and Education. Retrieved May 16, 2020, from https://jamesmccaffrey.wordpress.com/2006/04/28/validation-vs-verification/

Mure, G.R.G. (2007). Posterior Analytics. The University of Adelaide.

Postulate (2010, May 22). Oxford English Dictionary Retrieved May 10, 2020, from https://www.oxfordlearnersdictionaries.com/search/english/?q=postulate

Schein, L.E., \& Greiner, V.E. (1988). Power and organization development: mobilizing power to implement change (Repr. with corrections. ed.). Reading, Mass.: Addison-Wesley.

\section{Notes:}

[1] Sir Karl Raimund Popper (1902-1994) was an Austrian-born British philosopher, academic and social commentator. One of the 20th century's most influential philosophers of science, he is known for his rejection of the classical inductivist views on the scientific method in favour of empirical falsification.

\section{Appendix}

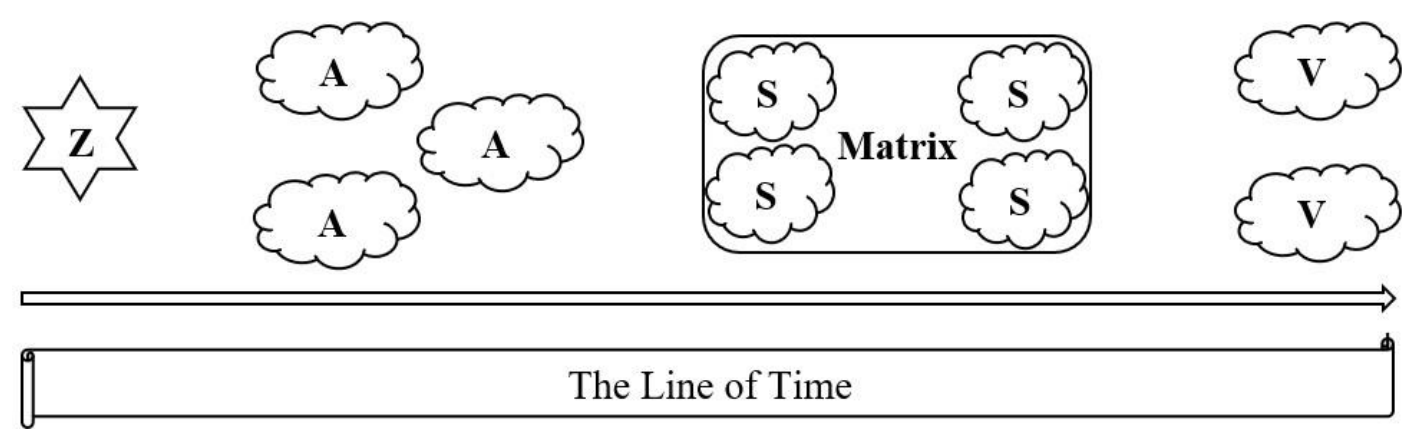

Figure 1. Relation of Quasar to Archions, the Matrix, and Versions in the schematic representation of modeling in the framework of the Theory 


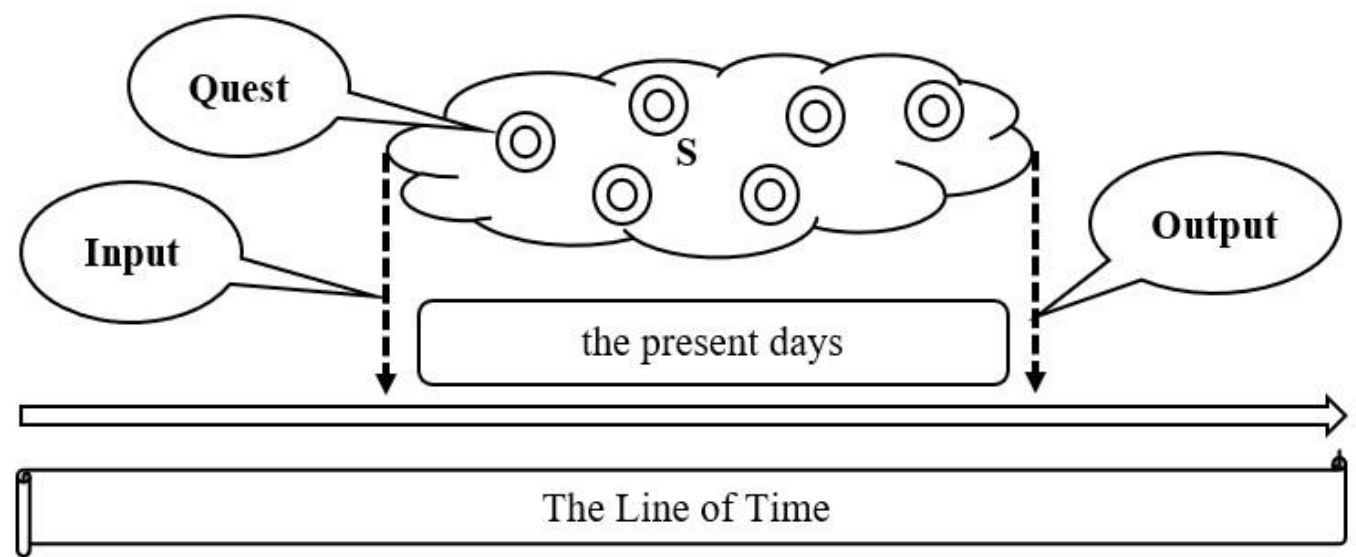

Figure 3. Formation of a set of Quests on the example of Station in the schematic representation of modeling in the framework of the Theory

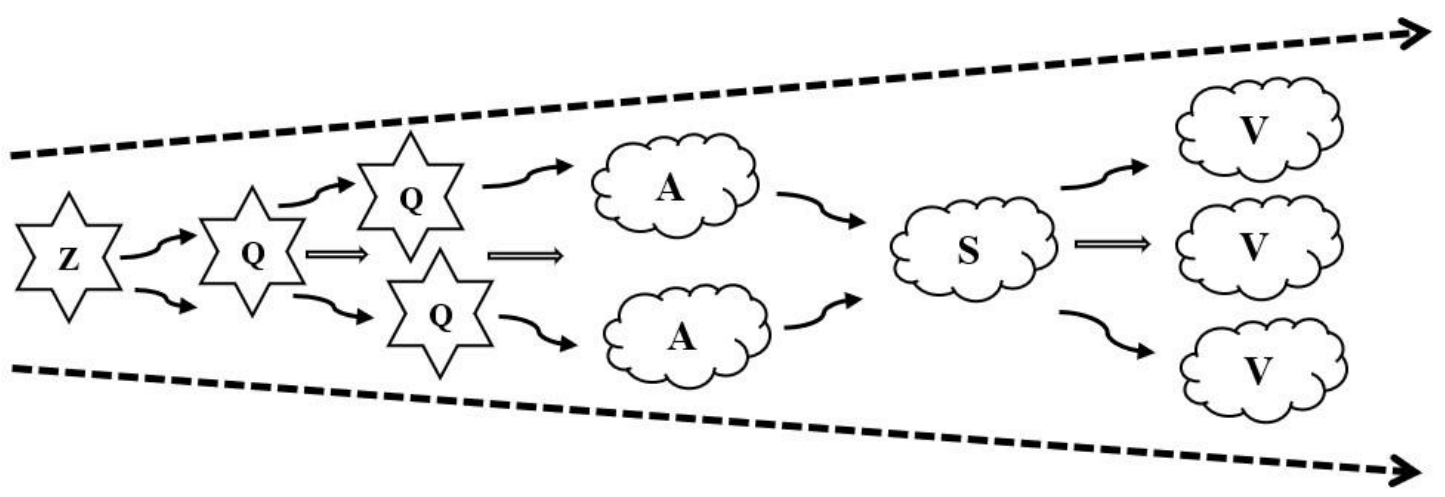

Figure 2. The formation and reflection of the Relict Background on the example of Version formation 